\title{
Soluble model of Bose-atoms with two level internal structure: non-conventional Bose-Einstein condensation
}

\author{
M. Corgin $112 ;$, D.P. Sankovich $\left.3\right|^{\dagger}$ \\ 1 Departamento de Matemáticas, Universidad de La Serena, Cisternas 1200, La Serena, Chile \\ 2 Laboratorio de Análisis Estocástico, Chile \\ 3 Steklov Mathematical Institute, Gubkin Str. 8, 119991, Moscow, Russia
}

Received July 21, 2010

\begin{abstract}
For a Bose atom system whose energy operator is diagonal in the so-called number operators and its ground state has an internal two-level structure with negative energies, exact expressions for the limit free canonical energy and pressure are obtained. The existence of non-conventional Bose-Einstein condensation has been also proved.
\end{abstract}

Key words: approximating Hamiltonian method, non-conventional Bose-Einstein condensation

PACS: 05.30.Jp, 67.85.Jk

\section{Introduction}

We use an approach based on a suitable expression obtained for the limit free canonical energy in order to determine the limit pressure of a Bose-atom system with internal two-level structure. This enables us to recover some results, related to non-conventional Bose-Einstein condensation (BEC), obtained in [1] in the framework of the approximating Hamiltonians method ([2]).

In section 2 we present a description of the main mathematical features associated with this model. In section 3 we obtain the limit free canonical energy of the model. It leads via Legendre transform to the limit pressure, recovering the previous results obtained in [1]. Finally in section 4 it is proved that the system undergoes non-conventional BEC (independent of temperature BEC).

\section{The model}

The one-particle free Hamiltonian corresponds to the operator $S^{l}=-\triangle / 2$ defined on a dense subset of the Hilbert space $\mathcal{H}^{l}=L^{2}\left(\Lambda_{l}\right)$, being $\Lambda_{l}=[-l / 2, l / 2]^{d} \subset \mathbb{R}^{d}$ a cubic box of boundary $\partial \Lambda_{l}$ and volume $V_{l}=l^{d}$. In other words, the particles are confined to bounded regions. We assume periodic boundary conditions under which $S^{l}$ becomes a self-adjoint operator.

We consider a system of Bose atoms with an internal two-level structure analogous to the SU2 spin symmetry. In this case any one-particle wave function has the form $\phi \otimes s$ where, $\phi \in L^{2}\left(\Lambda_{l}\right)$ and $s \in \mathbb{C}^{2}$ represents the internal state. Therefore, the vector space associated with this system is in fact, $\mathcal{H}_{s}^{l}=L^{2}\left(\Lambda_{l}\right) \otimes \mathbb{C}^{2}$.

We shall study a model of Bose particles whose Hamiltonian is given by:

$$
\hat{H}_{l}=\hat{H}_{l}^{0}+\frac{a}{V_{l}} \sum_{\mathbf{p} \in \Lambda_{l}^{*}, \sigma}\left(\hat{a}_{\mathbf{p}, \sigma}^{\dagger}\right)^{2} \hat{a}_{\mathbf{p}, \sigma}^{2}+\frac{\gamma}{V_{l}} \hat{n}_{\mathbf{0},-} \hat{n}_{\mathbf{0},+},
$$

\footnotetext{
*E-mail: mcorgini@userena.cl

${ }^{\dagger}$ E-mail: sankovch@mi.ras.ru
} 
where $\sigma=+$ or - depending on the corresponding internal level. The second term at the right hand side of equation (11) represents the intrastate collisions (self-scattering term), the third term represents the interstate collisions (cross-scattering term).

This model has been exhaustively studied in [1] by using the so-called method of approximating Hamiltonians developed in [2]. Here we shall obtain an analytical expression for the limit pressure of our model as the Legendre transform of the free canonical energy.

The sum in (11) runs over the set $\Lambda_{l}^{*}=\left\{\mathbf{p}=\left(p_{1}, \ldots, p_{d}\right) \in \mathbb{R}^{d}: p_{\alpha}=2 \pi n_{\alpha} / l, n_{\alpha} \in \mathbb{Z}, \alpha=\right.$ $1,2, \ldots, d\} . \hat{a}_{\mathbf{p}, \sigma}^{\dagger}, \hat{a}_{\mathbf{p}, \sigma}$ are the Bose operators of creation and annihilation of particles defined on the Bose Fock space $\mathcal{F}_{\mathrm{B}}$ and satisfying the usual commutation rules: $\left[\hat{a}_{\mathbf{q}, \sigma_{1}}, \hat{a}_{\mathbf{p}, \sigma_{2}}^{\dagger}\right]=\hat{a}_{\mathbf{q}, \sigma_{1}} \hat{a}_{\mathbf{p}, \sigma_{2}}^{\dagger}-$ $\hat{a}_{\mathbf{p}, \sigma_{2}}^{\dagger} \hat{a}_{\mathbf{q}, \sigma_{1}}=\delta_{\mathbf{p}, \mathbf{q}} \delta_{\sigma_{1}, \sigma_{2}} \cdot \hat{n}_{\mathbf{p}, \sigma}=\hat{a}_{\mathbf{p}, \sigma}^{\dagger} \hat{a}_{\mathbf{p}, \sigma}$ is the number operator associated with mode $\mathbf{p}$ and internal level $\sigma$. In this case $\hat{H}_{l}^{0}=\sum_{\mathbf{p} \in \Lambda_{l}^{*}, \sigma} \lambda_{l}(\mathbf{p}, \sigma) \hat{n}_{\mathbf{p}, \sigma}, a>0, \gamma \in \mathbb{R} \cdot \hat{N}=\sum_{\mathbf{p} \in \Lambda_{l}^{*}, \sigma} \hat{a}_{\mathbf{p}, \sigma}^{\dagger} \hat{a}_{\mathbf{p}, \sigma}$ is the total number operator, $\hat{N}^{\prime}=\sum_{\mathbf{p} \in \Lambda_{l}^{*} \backslash\{\mathbf{0}\}, \sigma} \hat{a}_{\mathbf{p}, \sigma}^{\dagger} \hat{a}_{\mathbf{p}, \sigma}$ is the total number operator with exclusion of $\hat{n}_{\mathbf{0},-}, \hat{n}_{\mathbf{0},+}$ and

$$
\lambda_{l}(\mathbf{p}, \sigma)= \begin{cases}\lambda_{l}(\mathbf{0}, \sigma)<0, & \mathbf{p}=\mathbf{0} \\ \mathbf{p}^{2} / 2, & \mathbf{p} \neq \mathbf{0}\end{cases}
$$

where $\lambda_{l}(\mathbf{0},-)=-\lambda-O\left(V_{l}^{-s}\right), \lambda_{l}(\mathbf{0},+)=-\lambda+O\left(V_{l}^{-s}\right)$ with $s>0, \lambda>0$ and $\lambda_{l}(\mathbf{0}, \sigma) \rightarrow$ $\lambda(\mathbf{0}, \sigma)=-\lambda$ as $V_{l} \rightarrow \infty$.

Note that the boson Fock space $\mathcal{F}_{\mathrm{B}}$ is isomorphic to the tensor product $\otimes_{\sigma, \mathbf{p} \in \Lambda_{l}^{*}} \mathcal{F}_{\mathbf{p}, \sigma}^{\mathrm{B}}$ where $\mathcal{F}_{\mathbf{p}, \sigma}^{\mathrm{B}}$ is the boson Fock space constructed on the one-dimensional Hilbert space $\mathcal{H}_{\mathbf{p}, \sigma}=\left\{\gamma \mathrm{e}^{i \mathbf{p} \cdot \mathbf{x}} \otimes e_{\sigma}\right\}_{\gamma \in \mathbb{C}}$ where $e_{-}=(0,1)$ and $e_{+}=(1,0)$.

Let

$$
p_{l}(\beta, \mu)=\frac{1}{\beta V_{l}} \ln \operatorname{Tr}_{\mathcal{F}_{\mathrm{B}}} \exp \left(-\beta\left(\hat{H}_{l}-\mu \hat{N}\right)\right)
$$

be the grand-canonical pressure, at finite volume, corresponding to $\hat{H}_{l}$, where $\beta=\theta^{-1}$ is the inverse temperature. as

If $\hat{H}_{l}(\mu)=\hat{H}_{l}-\mu \hat{N}$, the equilibrium Gibbs state (grand canonical ensemble) $\langle-\rangle_{\hat{H}_{l}(\mu)}$ is defined

$$
\langle\hat{A}\rangle_{\hat{H}_{l}(\mu)}=\left[\operatorname{Tr}_{\mathcal{F}_{\mathrm{B}}} \exp \left(-\beta \hat{H}_{l}(\mu)\right)\right]^{-1} \operatorname{Tr}_{\mathcal{F}_{\mathrm{B}}} \hat{A} \exp \left(-\beta \hat{H}_{l}(\mu)\right)
$$

for any operator $\hat{A}$ acting on the symmetric Fock space.

Finally, the total density of particles $\rho(\mu)$ for infinite volume is defined as

$$
\lim _{V_{l} \rightarrow \infty}\left\langle\frac{\hat{N}}{V_{l}}\right\rangle_{\hat{H}_{l}(\mu)}=\lim _{V_{l} \rightarrow \infty} \rho_{l}(\mu)=\rho(\mu),
$$

and the density of particles $\rho_{\mathbf{0}, \sigma}(\beta, \mu)$ associated with the energy $\lambda(\mathbf{0}, \sigma)=-\lambda$ is defined as

$$
\lim _{V_{l} \rightarrow \infty}\left\langle\frac{\hat{n}_{\mathbf{0}, \sigma}}{V_{l}}\right\rangle_{\hat{H}_{l}(\mu)}=\lim _{V_{l} \rightarrow \infty} \rho_{\mathbf{0}, \sigma, l}(\beta, \mu)=\rho_{\mathbf{0}, \sigma}(\beta, \mu) .
$$

We shall say that the system undergoes a macroscopic occupation of the single particle mode $(\mathbf{0}, \sigma)(\mathrm{BEC})$ if $\rho_{\mathbf{0}, \sigma}(\beta, \mu)>0$.

\section{Pressure}

Let $f_{l}(\beta, \varrho)$ be the free canonical energy at finite volume $V_{l}$, inverse temperature $\beta$ and density $\varrho$, corresponding to Hamiltonian given by equation (11). Let $\tilde{f}_{l}(\beta, \varrho), f_{l}^{\text {id }}(\beta, \varrho), f_{l}^{\text {id }^{\prime}}(\beta, \varrho)$ be the 
finite free canonical energies associated with $\tilde{H}_{l}, \hat{H}_{l}^{\mathrm{id}}$, and $\hat{H}_{l}^{\text {id }}{ }^{\prime}$, respectively. These operators are given by

$$
\tilde{H}_{l}=\hat{H}_{l}^{\mathrm{id}}+\frac{a}{V_{l}} \sum_{\mathbf{p} \in \Lambda_{l}^{*}, \sigma} \hat{n}_{\mathbf{p}, \sigma}^{2}+\frac{\gamma}{V_{l}} \hat{n}_{\mathbf{0},-} \hat{n}_{\mathbf{0},+},
$$

where

$$
\begin{gathered}
\hat{H}_{l}^{\mathrm{id}}=-\lambda\left(\hat{n}_{\mathbf{0},-}+\hat{n}_{\mathbf{0},+}\right)+\sum_{\mathbf{p} \in \Lambda_{l}^{*} \backslash\{\mathbf{0}\}, \sigma} \lambda_{l}(\mathbf{p}, \sigma) \hat{n}_{\mathbf{p}, \sigma}, \\
\hat{H}_{l}^{\mathrm{id}^{\prime}}=\sum_{\mathbf{p} \in \Lambda_{l}^{*} \backslash\{\mathbf{0}\}, \sigma} \lambda_{l}(\mathbf{p}, \sigma) \hat{n}_{\mathbf{p}, \sigma} .
\end{gathered}
$$

Let $f(\beta, \varrho), \tilde{f}(\beta, \varrho), f^{\mathrm{id}}(\beta, \varrho), f^{\mathrm{id}^{\prime}}(\beta, \varrho)$ be the corresponding limit free canonical energies. Let $\varrho_{l}=N / V_{l}, \varrho=\lim _{V_{l}, N \rightarrow \infty} \varrho_{l}=$ constant. We shall use the symbol $\varrho$ when referring to $\varrho_{l}$ or $\varrho$ indistinctively, avoiding excessive notation.

The strategy developed in [3] enables us to prove the following theorem.

\section{Theorem 1.}

$$
\tilde{f}(\beta, \varrho)=-\inf _{\varrho_{\mathbf{0},-}, \varrho_{\mathbf{0},+} \in[0, \varrho]}\left\{-\lambda\left(\varrho_{\mathbf{0},-}+\varrho_{\mathbf{0}+}\right)+a \varrho_{\mathbf{0},-}^{2}+a \varrho_{\mathbf{0},+}^{2}+\gamma \varrho_{\mathbf{0}}-\varrho_{\mathbf{0}+}+f^{\mathrm{id}^{\prime}}\left(\beta, \varrho-\varrho_{\mathbf{0}}\right)\right\} .
$$

Proof. Being $n_{\mathbf{p}, \sigma}=0,1,2, \ldots, \varrho_{\mathbf{0},-}=n_{\mathbf{0},-} / V_{l}, \varrho_{\mathbf{0},+}=n_{\mathbf{0},+} / V_{l}$, the finite canonical free energies $f_{l}^{\text {id }}(\beta, \varrho), \tilde{f}_{l}(\beta, \varrho)$, can be written in the following form,

$$
\begin{aligned}
f_{l}^{\mathrm{id}}(\beta, \varrho) & =-\frac{1}{\beta V_{l}} \ln \sum_{n_{\mathbf{p}, \sigma}=0,1,2, \ldots, \mathbf{p} \in \Lambda_{l}^{*}, \sigma} \exp \left(-\beta \sum \lambda_{l}(\mathbf{p}, \sigma) n_{\mathbf{p}, \sigma}\right) \delta_{\sum_{\mathbf{p} \in \Lambda_{l}^{*}, \sigma} n_{\mathbf{p}, \sigma}=\left[\varrho V_{l}\right]}, \\
\tilde{f}_{l}(\beta, \varrho) & =-\frac{1}{\beta V_{l}} \ln \left(\sum_{\ldots+n_{\mathbf{p}, \sigma}+\cdots=\left[\varrho V_{l}\right]} \mathrm{e}^{-\beta V_{l} h_{l}\left(\varrho, \varrho_{\mathbf{0},-}, \varrho \mathbf{o},+\right)}\right),
\end{aligned}
$$

where

$$
\begin{aligned}
& h_{l}\left(\varrho, \varrho_{\mathbf{0},-}, \varrho_{\mathbf{0},+}\right)=-\lambda\left(\varrho_{\mathbf{0},-}+\varrho_{\mathbf{0},+}\right)+\varrho_{\mathbf{0},+}^{2}+a \varrho_{\mathbf{0},+}^{2}+\gamma \varrho_{\mathbf{0},-} \varrho_{\mathbf{0},+} \\
& -\frac{1}{\beta V_{l}} \ln \sum_{n_{\mathbf{p}, \sigma}=0,1, \ldots, \mathbf{p} \in \Lambda_{l}^{*} \backslash\{\mathbf{0}\}, \sigma} \exp \left[-\beta\left(\sum \lambda_{l}(\mathbf{p}, \sigma) n_{\mathbf{p}, \sigma}+\frac{a}{V_{l}} n_{\mathbf{p}, \sigma}^{2}\right)\right] \delta_{N^{\prime}=\left[\varrho V_{l}\right]-\left[\varrho_{0} V_{l}\right]}
\end{aligned}
$$

and $N^{\prime}=\sum_{\mathbf{p} \in \Lambda_{l}^{*} \backslash\{\mathbf{0}\}, \sigma} n_{\mathbf{p}, \sigma}$. The following inequality

$$
\begin{aligned}
-\tilde{f}_{l}(\beta, \varrho) & =\frac{1}{\beta V_{l}} \ln \left(\sum_{. .+n_{\mathbf{p}, \sigma}+. .=\left[\varrho_{l}\right]} \mathrm{e}^{-\beta V_{l} h_{l}\left(\varrho, \varrho_{\mathbf{0},-}, \varrho_{\mathbf{0},+}\right)}\right) \geqslant \frac{1}{\beta V_{l}} \ln \left(\mathrm{e}^{-\beta V_{l} h_{l}\left(\varrho_{,} \varrho_{\mathbf{0},-}, \varrho_{\mathbf{0},+}\right)}\right) \\
& =-h_{l}\left(\varrho, \varrho_{\mathbf{0},-}, \varrho_{\mathbf{0},+}\right),
\end{aligned}
$$

holds for $\varrho_{\mathbf{0},-}, \varrho_{\mathbf{0},+} \in[0, \varrho]$, being $[b]$ the integer part of $b$. Equation (10) implies that,

$$
\tilde{f}_{l}(\beta, \varrho) \leqslant \inf _{\varrho_{0,-}, \varrho_{0,+} \in[0, \varrho]} h_{l}\left(\varrho, \varrho_{\mathbf{0},-}, \varrho_{\mathbf{0},+}\right)
$$


On the other hand, being $n_{\mathbf{0}}=n_{\mathbf{0},+}+n_{\mathbf{0},-}$, we have,

$$
\begin{aligned}
-\tilde{f}_{l}(\beta, \varrho) & \leqslant \frac{1}{\beta V_{l}} \ln \left(\sum_{n_{\mathbf{0}}=0, N^{\prime}=0}^{\left[\varrho V_{l}\right]} \exp \left(-\beta V_{l} \inf _{\varrho_{\mathbf{0},-}, \varrho_{\mathbf{0},+} \in[0, \varrho]} h_{l}\left(\varrho, \varrho_{\mathbf{0},-}, \varrho_{\mathbf{0},+}\right)\right)\right) \\
& \leqslant \frac{1}{\beta V_{l}} \ln \left(\exp \left(-\beta V_{l} \inf _{\varrho_{\mathbf{0},-}, \varrho_{\mathbf{0},+} \in[0, \varrho]} h_{l}\left(\varrho, \varrho_{\mathbf{0},-}, \varrho_{\mathbf{0},+}\right)\right) \sum_{n_{\mathbf{0}}=0, N^{\prime}=0}^{\left[\varrho V_{l}\right]} 1\right) \\
& \leqslant-\inf _{\varrho_{\mathbf{0},-}, \varrho_{\mathbf{0},+} \in[0, \varrho]} h_{l}\left(\varrho, \varrho_{\mathbf{0},-}, \varrho_{\mathbf{0},+}\right)+\frac{2}{\beta V_{l}} \ln \left(1+\frac{\left[\varrho V_{l}\right]\left(1+\left[\varrho V_{l}\right]\right)}{2}\right) \\
& \leqslant-\inf _{\varrho_{\mathbf{0},-}, \varrho_{\mathbf{0},+} \in[0, \varrho]} h_{l}\left(\varrho, \varrho_{\mathbf{0},-}, \varrho_{\mathbf{0},+}\right)+\frac{4}{\beta V_{l}} \ln \left(\left[\varrho V_{l}\right]+1\right) .
\end{aligned}
$$

Thus, we obtain the inequalities

$$
\inf _{\varrho_{\mathbf{0},-}, \varrho_{\mathbf{0},+} \in[0, \varrho]} h_{l}\left(\varrho, \varrho_{\mathbf{0},-}, \varrho_{\mathbf{0},+}\right)-\frac{4}{\beta V_{l}} \ln \left(\left[\varrho V_{l}\right]+1\right) \leqslant \tilde{f}_{l}(\beta, \varrho) \leqslant \inf _{\varrho_{\mathbf{0},-}, \varrho_{\mathbf{0},+} \in[0, \varrho]} h_{l}\left(\varrho, \varrho_{\mathbf{0},-}, \varrho_{\mathbf{0},+}\right) .
$$

Therefore, in the thermodynamic limit it follows that,

$$
\tilde{f}(\beta, \varrho)=\lim _{V_{l} \rightarrow \infty} \inf _{\varrho_{\mathbf{0},-}, \varrho_{\mathbf{0},+} \in[0, \varrho]} h_{l}\left(\varrho, \varrho_{\mathbf{0},-}, \varrho_{\mathbf{0},+}\right) .
$$

$h_{l}\left(\varrho, \varrho_{\mathbf{0},-}, \varrho_{\mathbf{0},+}\right)$ can be rewritten as

$$
\begin{aligned}
h_{l}\left(\varrho, \varrho_{\mathbf{0},-}, \varrho_{\mathbf{0},+}\right)= & -\lambda\left(\varrho_{\mathbf{0},-}+\varrho_{\mathbf{0},+}\right)+a \varrho_{\mathbf{0},-}^{2}+a \varrho_{\mathbf{0},+}^{2}+\gamma \varrho_{\mathbf{0},-} \varrho_{\mathbf{0},+} \\
& -\frac{1}{V_{l}} \ln \left\langle\exp \left(-\frac{\beta a}{V_{l}} \sum_{\mathbf{p} \in \Lambda_{l}^{*} \backslash\{\mathbf{0}\}, \sigma} \hat{n}_{\mathbf{p}, \sigma}^{2}\right)\right\rangle_{\hat{H}_{l}^{\mathrm{id}^{\prime}}\left(\beta, \varrho-\varrho_{\mathbf{0}}\right)}+f_{l}^{\mathrm{id}^{\prime}}\left(\beta, \varrho-\varrho_{\mathbf{0}}\right),
\end{aligned}
$$

being $\langle-\rangle_{\hat{H}_{l}^{\mathrm{id}}{ }_{\left(\beta, \varrho-\varrho_{0}\right)}}$ the canonical Gibbs state associated with $\hat{H}_{l}^{\mathrm{id}^{\prime}}\left(\beta, \varrho-\varrho_{0}\right)$. Since the limit free canonical energy of the free Bose gas is the Legendre transform of the corresponding pressure, we get,

$$
\lim _{V_{l} \rightarrow \infty} f_{l}^{\text {id }^{\prime}}(\beta, \varrho)=f^{\text {id }^{\prime}}(\beta, \varrho)=\sup _{\alpha \leqslant 0}\left\{\alpha \varrho-p^{\text {id }}(\beta, \alpha)\right\}
$$

being $p^{\mathrm{id}^{\prime}}(\beta, \alpha)$ the limit grand canonical pressure associated with $\hat{H}_{l}^{\mathrm{id}^{\prime}}$.

From the Jensen inequality we get

$$
\left\langle\exp \left(-\frac{\beta a}{V_{l}} \sum_{\mathbf{p} \in \Lambda_{l}^{*} \backslash\{\mathbf{0}\}, \sigma} \hat{n}_{\mathbf{p}, \sigma}^{2}\right)\right\rangle_{\hat{H}_{l}^{\mathrm{id}^{\prime}\left(\beta, \varrho-\varrho_{\mathbf{0}}\right)}} \geqslant \exp \left(-\beta a V_{l} \sum_{\mathbf{p} \in \Lambda_{l}^{*} \backslash\{\mathbf{0}\}, \sigma}\left\langle\frac{\hat{n}_{\mathbf{p}, \sigma}^{2}}{V_{l}^{2}}\right\rangle_{\hat{H}_{l}^{\mathrm{id}^{\prime}}\left(\beta, \varrho-\varrho_{\mathbf{0}}\right)}\right) .
$$

For $\mathbf{p}$ and $V_{l}$ fixed and $r \geqslant 1, r \in \mathbb{Z}^{+}$, the moments

$$
\left\langle\hat{n}_{\mathbf{p}, \sigma}^{r}\right\rangle_{\hat{H}_{l}^{\mathrm{id}^{\prime}}(\beta, \varrho-\varrho \mathbf{o})}
$$

in the canonical ensemble, are monotonously increasing functions of $\varrho$ (see [4, 5]). Therefore,

$$
\begin{aligned}
\left\langle\frac{\hat{n}_{\mathbf{p}, \sigma}^{2}}{V_{l}^{2}}\right\rangle_{\hat{H}_{l}^{\mathrm{id}^{\prime}}\left(\mu\left(\varrho-\varrho_{\mathbf{0}}\right)\right)} & \int_{[0, \infty)}\left\langle\frac{\hat{n}_{\mathbf{p}, \sigma}^{2}}{V_{l}^{2}}\right\rangle_{\hat{H}_{l}^{\mathrm{id}^{\prime}}(\beta, x)} \hat{\mathbb{K}}_{V_{l}}\left(\varrho-\varrho_{\mathbf{0}}, \mathrm{dx}\right) \\
& \geqslant \int_{\left[\varrho-\varrho_{\mathbf{0}}, \infty\right)}\left\langle\frac{\hat{n}_{\mathbf{p}, \sigma}^{2}}{V_{l}^{2}}\right\rangle_{\hat{H}_{l}^{\mathrm{id}^{\prime}}(\beta, x)} \hat{\mathbb{K}}_{V_{l}}\left(\varrho-\varrho_{\mathbf{0}}, \mathrm{dx}\right) \\
& \geqslant\left\langle\frac{\hat{n}_{\mathbf{p}, \sigma}^{2}}{V_{l}^{2}}\right\rangle_{\hat{H}_{l}^{\mathrm{id}^{\prime}}\left(\beta, \varrho-\varrho_{\mathbf{0}}\right)} \hat{\mathbb{K}}_{V_{l}}\left(\varrho-\varrho_{\mathbf{0}},\left[\varrho-\varrho_{\mathbf{0}}, \infty\right)\right),
\end{aligned}
$$


where $\langle\cdot\rangle_{\hat{H}_{l}^{\mathrm{id}}{ }^{\prime}(\mu)}$ is the Gibbs state associated with $\hat{H}_{l}^{\mathrm{id}{ }^{\prime}}$ in the grand-canonical ensemble given by equation (3) and $\hat{\mathbb{K}}_{V_{l}}\left(\varrho-\varrho_{0}, d x\right)$ is the so-called Kac measure of the perfect Bose gas at finite volume $V_{l}([4,[]])$ given by,

$$
\hat{\mathbb{K}}_{V_{l}}(\varrho, \mathrm{dx})=\delta(\mathrm{x}-\varrho) \mathrm{dx}
$$

for $\varrho \leqslant \varrho_{\mathrm{c}}(\beta)$, being $\varrho, \varrho_{\mathrm{c}}(\beta)=2(2 \pi)^{-d} \int_{\mathbb{R}^{d}}\left(\mathrm{e}^{\beta \mathbf{p}^{2} / 2}-1\right)^{-1} \mathrm{~d} \mathbf{p}$, the density and the critical density of the perfect Bose gas (the case of atoms with internal structure), respectively, and

$$
\hat{\mathbb{K}}_{V_{l}}(\varrho, \mathrm{d} x)= \begin{cases}0, & \text { for } x \leqslant \varrho_{\mathrm{c}}(\beta), \\ \left(\varrho-\varrho_{\mathrm{c}}(\beta)\right)^{-1} \exp \left(-\frac{x-\varrho_{\mathrm{c}}(\beta)}{\varrho-\varrho_{\mathrm{c}}(\beta)}\right) \mathrm{dx}, & \text { for } x>\varrho_{\mathrm{c}}(\beta)\end{cases}
$$

for $\varrho>\varrho_{\mathrm{c}}(\beta)$.

Using the latter inequality and taking into account that

$$
\begin{aligned}
\lim _{V_{l} \rightarrow \infty} \hat{\mathbb{K}}_{V_{l}}\left(\varrho-\varrho_{\mathbf{0}},\left[\varrho-\varrho_{\mathbf{0}}, \infty\right)\right) & \neq 0, \\
\lim _{V_{l} \rightarrow \infty} \sum_{\mathbf{p} \in \Lambda_{l}^{*} \backslash\{\mathbf{0}\}, \sigma}\left\langle\frac{\hat{n}_{\mathbf{p}, \sigma}^{2}}{V_{l}^{2}}\right\rangle_{\hat{H}_{l}^{\mathrm{id} \mathrm{d}^{\prime}}\left(\mu\left(\varrho-\varrho_{\mathbf{0}}\right)\right)} & =0,
\end{aligned}
$$

we get

$$
\lim _{V_{l} \rightarrow \infty} \sum_{\mathbf{p} \in \Lambda_{l}^{*} \backslash\{\mathbf{0}\}, \sigma}\left\langle\frac{\hat{n}_{\mathbf{p}, \sigma}^{2}}{V_{l}^{2}}\right\rangle_{\hat{H}_{l}^{\text {id' }}(\beta, \varrho-\varrho \mathbf{0})}=0 .
$$

Then, equations (15) and (17) imply that,

$$
\begin{aligned}
\lim _{V_{l} \rightarrow \infty} \inf _{\varrho_{\mathbf{0},-}, \varrho_{\mathbf{0}}+\in[0, \varrho]} h_{l}\left(\varrho, \varrho_{\mathbf{0},-}, \varrho_{\mathbf{0},+}\right) \leqslant & \inf _{\varrho_{\mathbf{0}-,} \varrho_{\mathbf{0}+} \in[0, \varrho]}\left\{-\lambda\left(\varrho_{\mathbf{0},-}+\varrho_{\mathbf{0},+}\right)\right. \\
& \left.+a \varrho_{\mathbf{0},+}^{2}+a \varrho_{\mathbf{0},+}^{2}+\gamma \varrho_{\mathbf{0},-} \varrho_{\mathbf{0},+}+f^{\mathrm{id}^{\prime}}\left(\beta, \varrho-\varrho_{\mathbf{0}}\right)\right\} .
\end{aligned}
$$

On the other hand, since $\exp \left\{-\beta a / V_{l} \sum_{\mathbf{p} \in \Lambda_{l}^{*} \backslash\{\mathbf{0}\}, \sigma} n_{\mathbf{p}, \sigma}^{2}\right\} \leqslant 1$ from equation (15) we get,

$$
\begin{aligned}
\lim _{V_{l} \rightarrow \infty} \inf _{\varrho_{\mathbf{0},-}, \varrho_{\mathbf{0},+} \in[0, \varrho]} h_{l}\left(\varrho, \varrho_{\mathbf{0},-}, \varrho_{\mathbf{0},+}\right) \geqslant & \lim _{V_{l} \rightarrow \infty \varrho_{\mathbf{0},-}, \inf _{\mathbf{0},+} \in[0, \varrho]}\left\{-\lambda\left(\varrho_{\mathbf{0},-}+\varrho_{\mathbf{0},+}\right)\right. \\
& \left.+a \varrho_{\mathbf{0},-}^{2}+a \varrho_{\mathbf{0},+}^{2}+\gamma \varrho_{\mathbf{0},-} \varrho_{\mathbf{0},+}+f_{l}^{\mathrm{id}^{\prime}}\left(\beta, \varrho-\varrho_{0}\right)\right\} .
\end{aligned}
$$

Equations (18), (19) imply

$$
\begin{aligned}
\lim _{V_{l} \rightarrow \infty} \inf _{\varrho_{\mathbf{0},-}, \varrho_{\mathbf{0},+} \in[0, \varrho]} h_{l}\left(\varrho, \varrho_{\mathbf{0},-}, \varrho_{\mathbf{0},+}\right)= & \lim _{V_{l} \rightarrow \infty} \inf _{\varrho_{\mathbf{0},-}, \varrho_{\mathbf{0},+} \in[0, \varrho]}\left\{-\lambda\left(\varrho_{\mathbf{0},-}+\varrho_{\mathbf{0},+}\right)\right. \\
& \left.+a \varrho_{\mathbf{0},-}^{2}+a \varrho_{\mathbf{0},+}^{2}+\gamma \varrho_{\mathbf{0},-} \varrho_{\mathbf{0},+}+f_{l}^{\mathrm{id}^{\prime}}\left(\beta, \varrho-\varrho_{0}\right)\right\} .
\end{aligned}
$$

\section{Theorem 2.}

$$
f(\beta, \varrho)=\tilde{f}(\beta, \varrho) .
$$

Proof. Let $\hat{H}_{l}^{(N)}$ and $\tilde{H}_{l}^{(N)}$ be the restrictions of the self-adjoint operators $\hat{H}_{l}$ and $\tilde{H}_{l}$ defined on $\mathcal{D} \subset \mathcal{F}_{\mathrm{B}}$ to the N-particles symmetrized Bose-space. In this case the following well-known Bogolyubov inequalities for free energies,

$$
\left\langle\frac{\Delta H_{l}^{(N)}}{V_{l}}\right\rangle_{\hat{H}_{l}^{(N)}(\varrho)} \leqslant f_{l}(\beta, \varrho)-\tilde{f}_{l}(\beta, \varrho) \leqslant\left\langle\frac{\Delta H_{l}^{(N)}}{V_{l}}\right\rangle_{\tilde{H}_{l}^{(N)}(\varrho)},
$$


hold, where $\Delta H_{l}^{(N)}=\hat{H}_{l}^{(N)}-\tilde{H}_{l}^{(N)},\langle-\rangle_{\hat{H}_{l}^{(N)}(\varrho)},\langle-\rangle_{\tilde{H}_{l}^{(N)}(\varrho)}$ are the Gibbs states in the canonical ensemble associated with the Hamiltonians $\hat{H}_{l}^{(N)}, \tilde{H}_{l}^{(N)}$, respectively.

Being $\Delta \hat{n}_{\mathbf{0}}=\hat{n}_{\mathbf{0},+}-\hat{n}_{\mathbf{0},-}$, this leads to the following inequalities,

$$
\begin{aligned}
& f_{l}(\beta, \varrho) \geqslant \tilde{f}_{l}(\beta, \varrho)-\frac{a}{V_{l}^{2}} \sum_{\mathbf{p}, \sigma}\left\langle\hat{n}_{\mathbf{p}, \sigma}\right\rangle_{\hat{H}_{l}^{(N)}(\varrho)}+\frac{O\left(V_{l}^{-s}\right)}{V_{l}}\left\langle\Delta \hat{n}_{\mathbf{0}}\right\rangle_{\hat{H}_{l}^{(N)}(\varrho)}, \\
& f_{l}(\beta, \varrho) \leqslant \tilde{f}_{l}(\beta, \varrho)-\frac{a}{V_{l}^{2}} \sum_{\mathbf{p}, \sigma}\left\langle\hat{n}_{\mathbf{p}, \sigma}\right\rangle_{\tilde{H}_{l}^{(N)}(\varrho)}+\frac{O\left(V_{l}^{-s}\right)}{V_{l}}\left\langle\Delta \hat{n}_{\mathbf{0}}\right\rangle_{\tilde{H}_{l}^{(N)}(\varrho)} .
\end{aligned}
$$

Noting that,

$$
\lim _{V_{l} \rightarrow \infty} \frac{1}{V_{l}^{2}} \sum_{\mathbf{p}, \sigma}\left\langle\hat{n}_{\mathbf{p}, \sigma}\right\rangle_{\hat{H}_{l}^{(N)}(\varrho)}=\lim _{V_{l} \rightarrow \infty} \frac{1}{V_{l}^{2}} \sum_{\mathbf{p}, \sigma}\left\langle\hat{n}_{\mathbf{p}, \sigma}\right\rangle_{\hat{H}_{l}^{(N)}(\varrho)}=0
$$

and

we obtain

$$
\lim _{V_{l} \rightarrow \infty} \frac{O\left(V_{l}^{-s}\right)}{V_{l}}\left\langle\Delta \hat{n}_{\mathbf{0}}\right\rangle_{\hat{H}_{l}^{(N)}(\varrho)}=\lim _{V_{l} \rightarrow \infty} \frac{O\left(V_{l}^{-s}\right)}{V_{l}}\left\langle\Delta \hat{n}_{\mathbf{0}}\right\rangle_{\tilde{H}_{l}^{(N)}(\varrho)}=0
$$

$$
\lim _{V_{l} \rightarrow \infty} f_{l}(\beta, \varrho)=\lim _{V_{l} \rightarrow \infty} \tilde{f}_{l}(\beta, \varrho)=\lim _{V_{l} \rightarrow \infty} \inf _{\varrho_{0},-, \varrho_{0}, \in[0, \varrho]} h_{l}\left(\varrho, \varrho_{0,-}, \varrho_{0,+}\right) .
$$

This completes the proof.

This result enables us to derive an explicit expression for the limit pressure $p(\beta, \mu)$ given by $p(\beta, \mu)=\lim _{V_{l} \rightarrow \infty} p_{l}(\beta, \mu)$

Let $q(x, y): \mathbb{R}^{2} \rightarrow \mathbb{R}$ be the symmetric quadratic form defined by,

$$
\begin{aligned}
& q(x, y)=(\mu+\lambda)(x+y)-a\left(x^{2}+y^{2}\right)-\gamma x y . \\
& \text { Let } \mathcal{A}=\left\{\mu \in \mathbb{R}: q^{*}=\sup _{x, y \in[0, \infty)} q(x, y)<\infty\right\} .
\end{aligned}
$$

Definition 1. The domain of stability $\mathcal{D}(p)$ of $p(\beta, \mu)$ is defined as,

$$
\mathcal{D}(p)=\left\{(\beta, \mu) \in \mathbb{R}^{2}: \beta>0, \mu \in \mathcal{A} \cap(-\infty, 0]\right\} .
$$

Corollary 1. For $(\beta, \mu) \in \mathcal{D}(p)$,

$$
p(\beta, \mu)=\sup _{\varrho_{\mathbf{0},-}, \varrho_{\mathbf{0},+} \in[0, \infty)}\left\{(\mu+\lambda)\left(\varrho_{\mathbf{0},-}+\varrho_{\mathbf{0},+}\right)-a\left(\varrho_{\mathbf{0},-}^{2}+\varrho_{\mathbf{0},+}^{2}\right)-\gamma \varrho_{\mathbf{0},-} \varrho_{\mathbf{0},+}\right\}+p^{\mathrm{id}^{\prime}}(\beta, \mu) .
$$

Proof. Since $f(\beta, \varrho)$ is a convex function of $\varrho$, its Legendre transform coincides with the grand canonical limit pressure $p(\beta, \mu)$, i.e.,

$$
p(\beta, \mu)=\sup _{\varrho \geqslant 0}\{\mu \varrho-f(\beta, \varrho)\}=\sup _{\varrho \geqslant 0}\{\mu \varrho-\tilde{f}(\beta, \varrho)\} .
$$

Therefore

$$
\begin{aligned}
& p(\beta, \mu)=\sup _{\varrho \geqslant 0}\left\{\mu \varrho-\inf _{\varrho_{\mathbf{0},-}, \varrho_{\mathbf{0},+} \in[0, \varrho]}\left\{-\lambda\left(\varrho_{\mathbf{0},-}+\varrho_{\mathbf{0},+}\right)+a\left(\varrho_{\mathbf{0},-}^{2}+\varrho_{\mathbf{0},+}^{2}\right)\right.\right. \\
& \left.+\gamma \varrho_{0},-\varrho_{0},++f^{\mathrm{id}}\left(\beta, \varrho-\varrho_{0}\right)\right\} \\
& =\sup _{\varrho \geqslant 0} \sup _{\varrho_{\mathbf{0},-}, \varrho_{\mathbf{0},+} \in[0, \varrho]}\left\{(\mu+\lambda)\left(\varrho_{\mathbf{0},-}+\varrho_{\mathbf{0},+}\right)-a \varrho_{\mathbf{0},-}^{2}-a \varrho_{\mathbf{0},+}^{2}\right. \\
& \left.-\gamma \varrho_{0},-\varrho_{\mathbf{0},+}-f^{\mathrm{id}^{\prime}}\left(\beta, \varrho-\varrho_{\mathbf{0}}\right)+\mu\left(\varrho-\varrho_{\mathbf{0}}\right\}\right\} \\
& =\sup _{\varrho_{\mathbf{0},-}, \varrho_{\mathbf{0},+} \in[0, \infty)}\left\{(\mu+\lambda)\left(\varrho_{\mathbf{0},-}+\varrho_{\mathbf{0},+}\right)-a\left(\varrho_{\mathbf{0},+}^{2}+\varrho_{\mathbf{0},+}^{2}\right)-\gamma \varrho_{\mathbf{0},-} \varrho_{\mathbf{0},+}\right\}+p^{\text {id }^{\prime}}(\beta, \mu) \text {. }
\end{aligned}
$$


This corollary implies that, the derivation of the limit pressure and demonstration of the occurrence of non-conventional Bose-Einstein condensation (independent of temperature) can be reduced to the study of the occurence of extreme values of the symmetric quadratic form $q$ given in equation (28).

Proposition 1. $q^{*}=\sup _{x, y \in[0, \infty)} q(x, y)$ satisfies $q^{*}=+\infty$, for $\gamma \in(-\infty,-2 a), \mu \in(-\lambda, 0]$.

$$
q^{*}= \begin{cases}0, & \mu \in(-\infty,-\lambda], \\ +\infty, & \mu \in(-\lambda, 0],\end{cases}
$$

for $\gamma=-2 a$.

$$
q^{*}= \begin{cases}0, & \mu \in(-\infty,-\lambda], \\ \frac{(\mu+\lambda)^{2}}{2 a+\gamma}, & \mu \in(-\lambda, 0],\end{cases}
$$

for $\gamma \in(-2 a, 2 a)$.

$$
q^{*}= \begin{cases}0, & \mu \in(-\infty,-\lambda], \\ \frac{(\mu+\lambda)^{2}}{4 a}, & \mu \in(-\lambda, 0],\end{cases}
$$

for $\gamma \in[2 a, \infty)$.

Proof. Let us introduce some basic notions concerning minimization and maximization of convex and concave quadratic functions. Let $f: \mathbb{R}^{n} \rightarrow \mathbb{R}$ be the quadratic form given by:

$$
f(\mathbf{x})=\frac{1}{2} \mathbf{x} Q \mathbf{x}^{\mathrm{T}}+\mathbf{c} \mathbf{x}^{\mathrm{T}},
$$

where $Q$ is a symmetric $n \times n$ - matrix of real entries and $\mathbf{c} \in \mathbb{R}^{n}$. The function $f$ is a convex (concave, respectively) function if and only if it is a symmetric and positive (negative,respectively) semidefinite function, i.e. $\mathbf{x} Q \mathbf{x}^{\mathrm{T}} \geqslant 0,\left(\mathbf{x} Q \mathbf{x}^{\mathrm{T}} \leqslant 0\right.$, respectively) for all $\mathbf{x} \in \mathbb{R}^{n}$. Then, being $f$ a convex (concave, respectively) function it attains its global minimum (global maximum, respectively) at $\mathbf{x}^{*}$ if and only if $\mathbf{x}^{*}$ solves the equations system $\nabla f(\mathbf{x})=Q \mathbf{x}^{\mathrm{T}}+\mathbf{c}=\mathbf{0}$. In this case the Hessian matrix $\mathcal{H}(\mathbf{x})$ satisfies $\mathcal{H}(\mathbf{x})=Q$.

For the quadratic form $q(x, y)$ given by equation (28) we have

$$
Q=-\left(\begin{array}{cc}
2 a & \gamma \\
\gamma & 2 a
\end{array}\right), \quad \mathbf{c}=(\mu+\lambda)(1,1)
$$

Therefore, $q$ is a strictly concave function $\left(\mathbf{x} Q \mathbf{x}^{\mathrm{T}}<0\right)$ if $a, \gamma$ satisfy the following condition:

$$
\operatorname{det} Q=\left|\begin{array}{cc}
2 a & \gamma \\
\gamma & 2 a
\end{array}\right|=-4 a^{2}+\gamma^{2}<0,
$$

i.e., $\gamma \in(-2 a, 2 a)$, and it is a strictly convex function $\left(\mathbf{x} Q \mathbf{x}^{\mathrm{T}}>0\right)$ if

$$
\operatorname{det} Q=-4 a^{2}+\gamma^{2}>0,
$$

i.e., $\gamma \in(-\infty,-2 a) \cup(2 a, \infty)$.

The same results can be obtained by using the standard approach based on second derivatives to study the functions of two variables.

We define the auxiliary quadratic forms $q_{1}, q_{2}, q_{3}$ by

$$
\begin{aligned}
& q_{1}(x, y)=(\mu+\lambda)(x+y)-a(x-y)^{2}, \\
& q_{2}(x, y)=(\mu+\lambda)(x+y)-a(x+y)^{2}, \\
& q_{3}(x, y)=(\mu+\lambda)(x+y)-\frac{\gamma}{2}(x+y)^{2} .
\end{aligned}
$$


a) Case $\mu \in(-\infty,-\lambda](\mu+\lambda \leqslant 0)$.

For $\mu$ satisfying the condition above we have, $q_{1}(x, y) \leqslant 0, q_{2}(x, y) \leqslant 0$ for all $x, y \in[0, \infty)$ and $\sup _{x, y \in[0, \infty)} q_{1}(x, y)=\sup _{x, y \in[0, \infty)} q_{2}(x, y)=0$. On the other hand, for $\gamma>0, q_{3}(x, y) \leqslant 0$ for all $x, y \in[0, \infty)$ and $\sup _{x, y \in[0, \infty)} q_{3}(x, y)=0$.

If $\gamma \in(-\infty,-2 a)$ and $x \in[0, \infty)$, the following inequality holds

$$
q^{*} \geqslant q(x, x)=(\mu+\lambda) 2 x-(2 a+\gamma) x^{2} .
$$

Since $-(2 a+\gamma)>0$, we get $\lim _{x \rightarrow+\infty} q(x, x)=+\infty$. Then, $q^{*}=+\infty$.

If $\gamma=-2 a$ and $x, y \in[0, \infty)$, we have $q(x, y)=q_{1}(x, y)$. Then $q *=0$.

If $\gamma \in(-2 a, 2 a)$ and $x, y \in[0, \infty)$, the following inequalities,

$$
q_{2}(x, y) \leqslant q(x, y) \leqslant q_{1}(x, y)
$$

hold, leading to the conclusion that $q^{*}=0$.

For $\gamma \in[2 a,+\infty)$ and $x, y \in[0, \infty)$ we obtain,

$$
q_{3}(x, y) \leqslant q(x, y) \leqslant q_{2}(x, y),
$$

which finally implies that $q^{*}=0$.

b) Case $\mu \in(-\lambda, 0](\mu+\lambda>0)$.

In this case, for $\gamma \in(-2 a, 2 a), q$ is a concave function, taking a global maximum at $x^{*}=$ $y^{*}=(\mu+\lambda) /(2 a+\gamma)$, for $(x, y) \in[0, \infty) \times[0, \infty)$. Then, using these results and equation (28) we get $q *=(\mu+\lambda)^{2} /(2 a+\gamma)$.

For $\gamma \in(-\infty,-2 a], x \in[0, \infty)$, taking into account that $\mu+\lambda>0,-(2 a+\gamma)>0$, we have,

$$
q^{*} \geqslant q(x, x)=\left(\mu+\lambda^{2}\right) 2 x-(2 a+\gamma) x^{2} \geqslant 0 .
$$

Then, noting that $\lim _{x \rightarrow+\infty} q(x, x)=+\infty$, we obtain $q^{*}=+\infty$.

For $\gamma \in[2 a, \infty)$ and $x, y \in[0, \infty)$, the following inequality holds,

$$
q(x, y) \leqslant q_{2}(x, y) .
$$

It is not hard to see that, $\sup _{x, y \in[0, \infty)} q_{2}(x, y)=(\mu+\lambda)^{2} / 4 a$, i.e.,

$$
q^{*} \leqslant \frac{(\mu+\lambda)^{2}}{4 a}
$$

Since for $x^{*}=(\mu+\lambda) / 2 a, y^{*}=0$ the quadratic form $q$ satisfies $q\left(x^{*}, 0\right)=(\mu+\lambda)^{2} / 4 a$, we conclude that $q^{*}=(\mu+\lambda)^{2} / 4 a$.

The above results completely determine $\mathcal{D}(p)$, and lead to the following theorem.

Theorem 3. For $(\beta, \mu) \in \mathcal{D}(p)$ the limit pressure is given by

$$
p(\beta, \mu)=q^{*}+p^{\operatorname{id}^{\prime}}(\beta, \mu) .
$$




\section{Non-conventional BEC}

From theorem 3 one easily deduces the following corollary on non-conventional BEC.

Corollary 2. Let $\rho_{\mathbf{0}}(\mu)=\rho_{\mathbf{0},-}(\mu)+\rho_{\mathbf{0},+}(\mu)$ be the total amount of condensate.

i.) For $\mu \in(-\infty,-\lambda]$,

$$
\rho_{\mathbf{0}}(\mu)=0
$$

if $\gamma \in[-2 a, \infty)$.

ii.) For $\mu \in(-\lambda, 0]$,

$$
\rho_{\mathbf{0}}(\mu)= \begin{cases}\frac{2(\mu+\lambda)}{2 a+\gamma}, & \gamma \in(-2 a, 2 a) \\ \frac{2(\mu+\lambda)}{4 a}, & \gamma \in[2 a, \infty)\end{cases}
$$

Non-conventional BEC takes place only for $\mu \in(-\lambda, 0]$ provided that $(\beta, \mu) \in \mathcal{D}(p)$. Moreover, the term representing interstate collisions satisfies:

$$
\lim _{V_{l} \rightarrow \infty}\left\langle\frac{\hat{n}_{\mathbf{0},-} \hat{n}_{\mathbf{0},+}}{V_{l}^{2}}\right\rangle_{\hat{H}_{l}(\mu)}=\left\{\begin{array}{cc}
\left(\frac{\mu+\lambda}{2 a+\gamma}\right)^{2}, & \gamma \in(-2 a, 2 a) \\
0, & \gamma \in[2 a, \infty)
\end{array}\right.
$$

For $\mu, \lambda$ fixed, with $\mu \in(-\lambda, 0]$, the total amount of condensate

$$
\rho_{\mathbf{0}}:(-2 a,+\infty) \rightarrow\left[\frac{\mu+\lambda}{2 a},+\infty\right)
$$

as function of $\gamma$ is a decreasing function, satisfying $\lim _{\gamma \downarrow-2 a} \rho_{\mathbf{0}}=+\infty, \lim _{\gamma \uparrow 2 a} \rho_{\mathbf{0}}=(\mu+\lambda) / 2 a$ and taking the constant value $(\mu+\lambda) / 2 a$ when $\gamma \in[2 a,+\infty)$. This behavior is a direct consequence of the fact that the symmetric quadratic form $q$ is a strictly concave function for $\gamma \in(-2 a, 2 a)$ and becomes a convex function for $\gamma \in(-\infty,-2 a) \cup(2 a,+\infty)$.

Hamiltonian (1) can be rewritten in the following form,

$$
\begin{aligned}
\hat{H}_{l}= & \sum_{\mathbf{p} \in \Lambda_{l}^{*}, \sigma} \lambda_{l}(\mathbf{p}, \sigma) \hat{n}_{\mathbf{p}, \sigma}+\left(\frac{2 a+\gamma}{2 V_{l}}\right)\left(\hat{n}_{\mathbf{0},-}^{2}+\hat{n}_{\mathbf{0},+}^{2}\right) \\
& -\frac{\gamma}{2 V_{l}}\left(\hat{n}_{\mathbf{0},-}-\hat{n}_{\mathbf{0},+}\right)^{2}-\frac{a}{V_{l}}\left(\hat{n}_{\mathbf{0},-}+\hat{n}_{\mathbf{0},+}\right)+\frac{a}{V_{l}} \sum_{\mathbf{p} \in \Lambda_{l}^{*} \backslash\{\mathbf{0}\}, \sigma}\left(\hat{a}_{\mathbf{p}, \sigma}^{\dagger}\right)^{2} \hat{a}_{\mathbf{p}, \sigma}^{2} .
\end{aligned}
$$

For $\mu \in(-\lambda, 0]$ and $\gamma \in(-2 a, 2 a)$, in the thermodynamic limit, only the term

$$
\left(\frac{2 a+\gamma}{2 V_{l}}\right)\left(\hat{n}_{\mathbf{0},-}^{2}+\hat{n}_{\mathbf{0},+}^{2}\right)
$$

contributes to the emergence of non-conventional BEC. In this sense, under those restrictions, our model is thermodynamically equivalent to the system whose energy is represented by the Hamiltonian,

$$
\hat{H}_{l}^{(1)}=\sum_{\mathbf{p} \in \Lambda_{l}^{*}, \sigma} \lambda_{l}(\mathbf{p}, \sigma) \hat{n}_{\mathbf{p}, \sigma}+\left(\frac{2 a+\gamma}{2 V_{l}}\right)\left(\hat{n}_{\mathbf{0},-}^{2}+\hat{n}_{\mathbf{0},+}^{2}\right)+\frac{a}{V_{l}} \sum_{\mathbf{p} \in \Lambda_{l}^{*} \backslash\{\mathbf{0}\}, \sigma}\left(\hat{a}_{\mathbf{p}, \sigma}^{\dagger}\right)^{2} \hat{a}_{\mathbf{p}, \sigma}^{2}
$$

On the other hand, for $\mu \in(-\lambda, 0]$ and $\gamma \in[2 a,+\infty)$, the model under study is thermodynamically equivalent to the model given by the following Hamiltonian,

$$
\hat{H}_{l}^{(2)}=\sum_{\mathbf{p} \in \Lambda_{l}^{*}, \sigma} \lambda_{l}(\mathbf{p}, \sigma) \hat{n}_{\mathbf{p}, \sigma}+\frac{a}{V_{l}}\left(\hat{n}_{\mathbf{0},-}^{2}+\hat{n}_{\mathbf{0},+}^{2}\right)+\frac{a}{V_{l}} \sum_{\mathbf{p} \in \Lambda_{l}^{*} \backslash\{\mathbf{0}\}, \sigma}\left(\hat{a}_{\mathbf{p}, \sigma}^{\dagger}\right)^{2} \hat{a}_{\mathbf{p}, \sigma}^{2} .
$$


Quantum many-particle systems associated with energy operators given by equations (35) and (36) have been extensively studied in [1, 7, 9] and references therein.

Adapting results in [6], it is easy to verify that for $d>2$, the models of the type given by equations (35) and (36) undergo generalized BEC in the following sense,

$$
\lim _{\delta \rightarrow 0^{+}} \lim _{V_{l} \rightarrow \infty} \sum_{\left\{\mathbf{p} \in \Lambda_{l}^{*}: 0<\|\mathbf{p}\|<\delta, \sigma= \pm\right\}}\left\langle\frac{\hat{n}_{\mathbf{0}, \sigma}}{V_{l}}\right\rangle_{\hat{H}_{l}(\mu)}= \begin{cases}0, & \rho \leqslant \rho_{\mathrm{c}}^{\mathrm{P}}(\beta), \\ \rho-\rho_{\mathrm{c}}^{\mathrm{P}}(\beta), & \rho>\rho_{\mathrm{c}}^{\mathrm{P}}(\beta)\end{cases}
$$

where,

$$
\rho_{\mathrm{c}}^{\mathrm{P}}(\beta)=\frac{2}{(2 \pi)^{d}} \int_{\mathbb{R}^{d}}\left(\mathrm{e}^{\beta \frac{\mathbf{p}^{2}}{2}}-1\right)^{-1} \mathrm{~d} \mathbf{p}
$$

is the critical density of the perfect Bose gas (Bose-atoms with internal two-level structure).

In this sense, for the model under study, conventional condensate coexists at $\mu=0$ with the non-conventional condensate (see [6]).

From a physical point of view the above facts imply that for $\gamma \in[2 a,+\infty)$, the interstate collisions term does not play any role in the thermodynamic behavior of the system and nonconventional BEC is only the consequence of the presence of intrastate collisions (self-scattering term). However, for $\gamma \in(-2 a, 2 a)$, non-conventional BEC is enhanced by the presence of a crossscattering term (for example, the case of a cross-scattering term with a negative coupling parameter $\gamma$ close to $-2 a)$.

\section{Conclusion}

We have made use of a strategy based on the derivation of the limit free canonical energy (see [3]) to obtain an analytic expression for the limit pressure of a system of Bose atoms whose ground state has two internal levels. We have proved that negative ground state energies, for a range of values of the chemical potential, leads to non-conventional BEC, being the amount of condensate as a function depending on the variables $\gamma, a$ associated with the interstate collisions and intrastate collisions. In this way we recover the previous results obtained in the framework of the approximating Hamiltonians method [1].

\section{Acknowledgements}

Partial financial support by PBCT-ACT13 (Stochastic Analysis Laboratory, Chile) and Programa de Magíster en Matemáticas, Universidad de La Serena, Chile.

\section{References}

1. Corgini M., Rojas-Molina C., Sankovich D.P., Int. J. Mod. Phys. B, 2008, 22, 4799-4815.

2. Bogolyubov N.N, Lectures on Quantum Statistics: Quasiaverages, Vol. 2, Gordon and Beach, New York, 1970.

3. Lewis J.T., Mark Kac Seminar on Probability and Physics: The Large Deviation Principle in Statistical Mechanics, syllabus 17, Centrum voor Wiskunde en Informatica (1985-1987) (Amsterdam: Centrum voor Wiskunde en Informatica CWI).

4. Pulé J.V., Zagrebnov V.A., J. Math. Phys., 2004, 268, 3565-3583.

5. Buffet E., Pulé J.V., J. Math. Phys., 1983, 24, 1608-1616.

6. Bru J.V., Nachtergaele B., Zagrebnov V.A., J. Stat. Phys., 2002, 109, 143-176.

7. Zagrebnov V.A., Condens. Matter Phys., 2000, 3, 265-275.

8. Corgini M., Sankovich D.P., Phys. Lett. A, 2007, 360, 419-422.

9. Bru J.B., Zagrebnov V.A., J. Phys. A: Math. Gen., 2000, 33, 449-464. 


\title{
Розв'язна модель Бозе-атомів 3 дворівневою внутрішньою структурою: нестандартна Бозе-Айнштайнівська конденсація
}

\author{
М. Коргін 112, д.П. Санкові ${ }^{3}$
}

1 Факультет математики, Університет де Ла Серена, Ла Серена, Чилі

2 Лабораторія стохастичного аналізу, Чилі

3 Математичний інститут ім. В.А. Стєклова, Москва, Росія

Для системи Бозе-атомів, чий оператор енергії є діагональним по так званому числу операторів і його основний стан має внутрішню дворівневу структуру з негативними енергіями, отримано точні вирази для граничних вільної канонічної енергії та тиску. Також доведено існування нестандартної Бозе-Айнштайнівська конденсації.

Ключові слова: метод апроксимуючого гамільтоніана, нестандартна Бозе-Айнштайнівська конденсація 
